Derein" gepflogenen Exörterungen geeignet exidbeint, ben mannig= fachen 2urforberungen bes Srivatforftoienftes zu entipredben.

2. Cine näbere Fühlung zmijhen ben einzelnen 2Ynwärtern bieję Dienftes zu juaffen, jet es zur biref́ten Stellenpermittlung, jei es zum 2 Uustautio von Stellungen, wie er infolge örtlicber, perjönltiber u. a. Berbältnifje nidyt jelten bet mandemen Stellen= inthaber rege wiro.

3reeds Rambaftmachung geetgneter Beamten bitten wix alle Forft= uno Sagobejitien im Sntereffe ber gatten Sadie utm gefl. Mit= teilung won neut zu bejesenden Stellen, jei es hinfichtlich gröperen ober fleineren SWirfungstreifes. Sbenjo werben alle, weldhe ben obenerwähnten

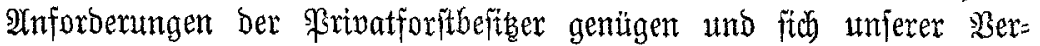

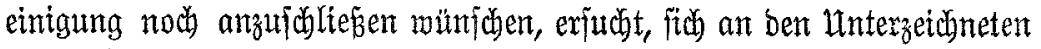
zit wertoen.

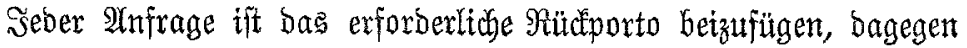
werben Seiträge nicht erboben.

(G) $\mathfrak{x} \mathfrak{B} \mathfrak{f} \mathfrak{a} \mathfrak{r} \mathfrak{x} \mathfrak{i}$. Thür., Den 15 . Februar 1903.

Mit $\mathfrak{B e i s m a n n}$ bheil!

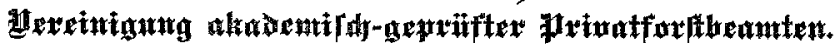

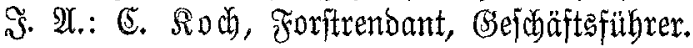

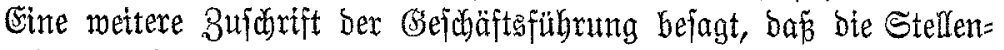
vermittlung nidft alleiniger Endzwed ber Bereinigung jein folle, fondern bejonderes Bsmicht anf bie anzuftrebende näbere Fühlung ber forft= beamten und bie badurch ermögliçte Förberung gemeinfamer šntereffen

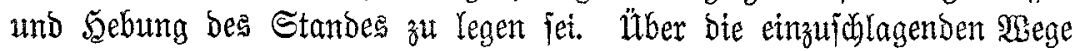
und Den weiteren 2 Uusbaut ber $\mathfrak{B}$ ereinigung foll eine nach Beenbigung

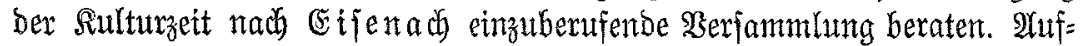
nabme in bie Sereinigung fönmen vorläntftg mtr biejentgent finden, welde fich einer afademifchen \$rüfung unterzagen haben, während ïber eventuelle

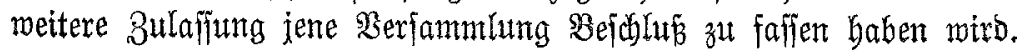

\section{Gitexatildye fexidgte.}

Iir. 28.

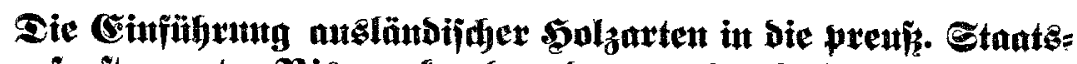

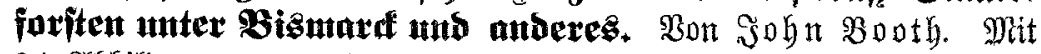

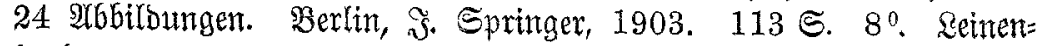
bants $5 \mathscr{M}$. 


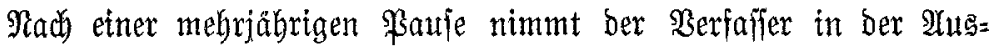
länoerfrage wieder das MBort. Err hat fich nach Der cinleitung biesmal bie $\mathfrak{A}$ ufgabe geftell:

1. Die Stellung bes Finriten Bismarct zu bejerer Frage unb Deffen

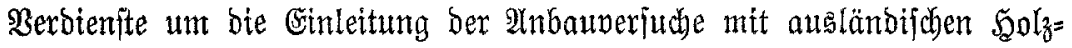
arten zu ichilloem,

2. $\mathfrak{2}$ n einigen $\mathfrak{S g l}_{z}$ arten, die fid bejonders bewährt haben, ben

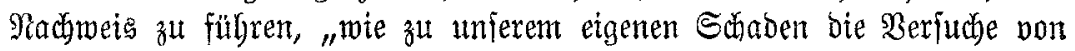
ben maß̧gebenton \$erjönlichleiten niemals geförbert feien" uno

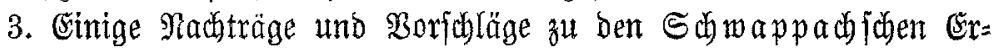
gebniffen zu beiprechent.

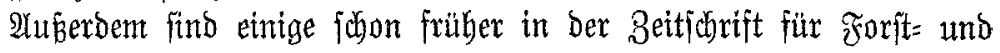

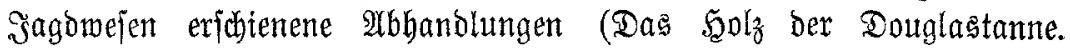

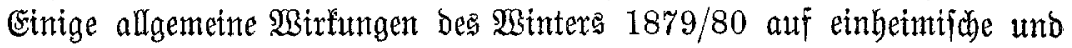

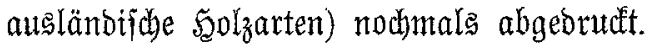

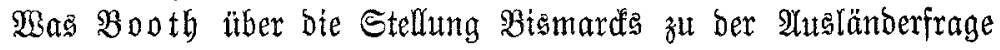
mitteilt, mird jeben Froritmann lebhaft interejifteren, namentlids wenn wir

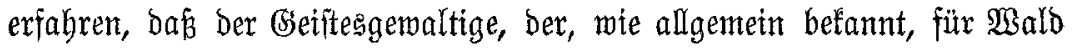
und Baldomirtichaft thets eine grop̉e Borliebe befundet hat, ben aus=

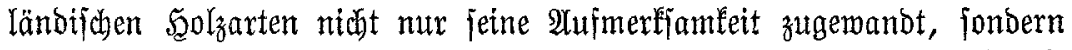
gerabezu den veriudbsmeifen 2lnbaut berjelben in Frettéen uno bamit mittelbar audh in anderen beutichen Etaaten im sabre 1880 amtlid)

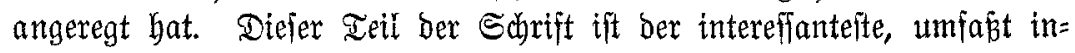
beffen nur 12 Seiten. $3 \mathfrak{u}$ bemjelben gebört bem inneren $3 \mathfrak{H}$ fammen= bange nach bie an anderer Stelle bes Buches bem Mortlaute nach mit= geteilte Denfichrift, bie Booth in Dem oben gebachten Sahre Dem F̧üriten

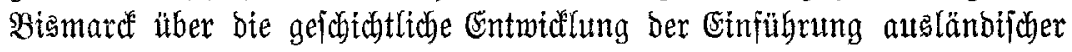
Solzarten in Eutropa eritattet hat, um befien 2 unfmerffamfeit auf biejen Begenftano zu lenfen; Dem mit der Sache, bez̧w. mit ben früheren

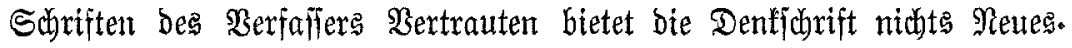

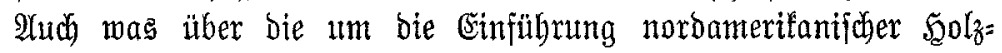

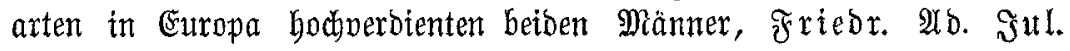

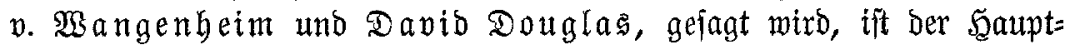
fache nad, namentlid was erfteren angeht, eine $\mathfrak{B i e b e r b o l u n g}$ befannter, von Booth jelbjt bereits hinreichend exörterter $\mathfrak{B e r h a ̈ l t n i f f e . ~}$

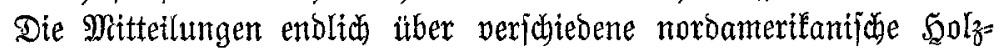

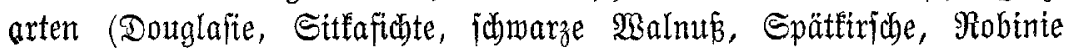
n. 1. w.) enthalten ebenfalls feine Bereiderung unjerer Renntnits von ber

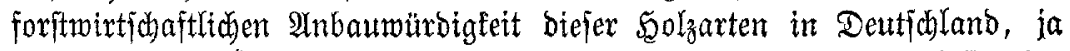

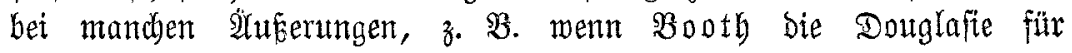


Riefernböben felbft ber 4. uno 5. Rlaffe empfteblt, nermag man tich eines Sopffichüttelnts ob folchen Urteils nityt zu ermebren.

Dabei berübrt in bohem Make unangenelgm bie aus ben früheren

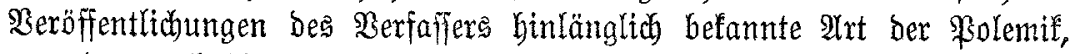
von ber auch bie vorltegende Shrift leiber wieberum Beugnis ablegt. und bie fich biesmal vorzugsmeife gegen bie, wie $\$$ booth glaubt, in ge=

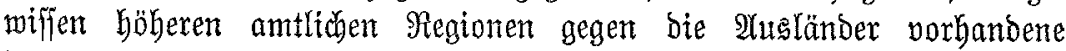
Dppofitton, fowie gegen biejenigen Bertreter ber Foritbotanif richtet, weldhe

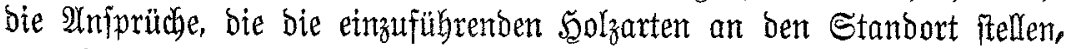
zu erforfichen und Danach Der Braries für beren Mnbaut in Deutficbland Fingerzeige zu geben in bantensmerter Meife bemüht find.

Wenngleich wir willig bie Berbiente, die $\mathfrak{B} 0$ oth fith burch die

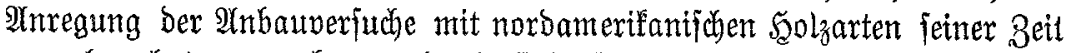
erworben bat, anzuerfennen bereit find, fo muz boch andrerfeits bie pon ihm mun fibn jett Sahbren beltebte Rampfesweife, bie mit einer wiffen= ichaftichen und retn fachlichen Förberung bes Begenitandes nichts gemein hat, mit aller Entjojiedenbeit zurüdgemiejen merben.

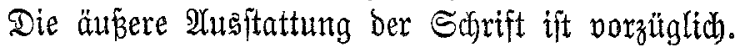

G.

\section{Mr. 29.}

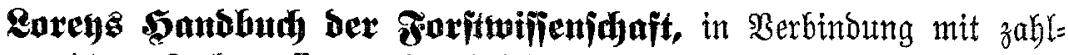
reidjen Fachgenoffen und Gelehrten berauggegeben non \$rofeffor

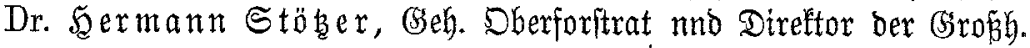

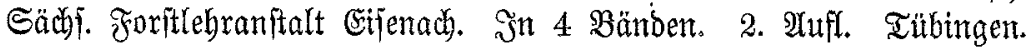

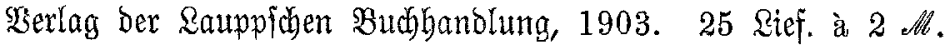

sm Sabr 1888 eridien unter Frofeffor Dr. Eoreys Redaftion bie erite Aluflage bes Şanbbuthes ber Foritmiffenfchaft, bie es fich zur Whifgabe ftellte, eine gebrängte Daritellung bes heutigen Standes der ge= jamten Foritwiffenichaft in initematijher 2ntoronung uno unter $B e=$ arbeitung ber einzelnen 2xbjhnitte burd Spezialifiten auf bent be= treffenden Gebiete zu geben. 2 Lußser Dem Şerauggeber felbit maren noch 16 Mitarbeiter, Durchaus in ber forfitichen Riteratur befannte Namen, an Dem Merf beteiligt. Dasfelbe hatte fich feitens ber Rrttif wie bes

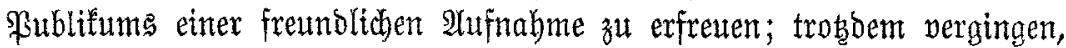
wie bies bei einem größzeren und barum aud foftfpieligeren Wexf nicht zu vermundern, 15 sahre, bis bie jethige zmeite, vermehrte und vielfach neubearbeitete 2 Ufflage ins seben trat.

Reiber war es bem verbienittollen Šerausgeber ber 1. Iltflage, bem alleits howgefwäksten Frofeffor Dr. Zvrey nidbt vergönnt, bie von ihm 
johon fräftig in IUngriff genommene Reutauflage zu erleben - ein jäber Iod ris ben in voller Mantestraft Stehenden mitten aus reicher und vielfeitiger Iätigfeit, und ein Mitarbeiter, Sberforftrat Dr. Stöber

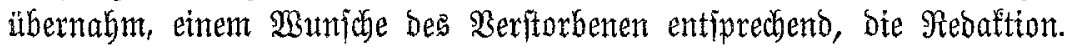

Sor uns liegen mun bie 4 erften steferungen, jebe bie eriten 6 Bogen eines ber 4 Bände umfaifient, in welchen bas garze $\mathfrak{B e r t}$ ex=

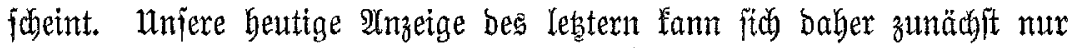

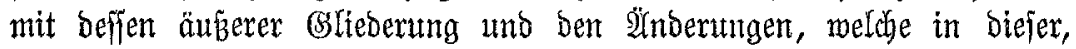
wie in ben Mitarbeitern eingetreten find, befafien.

Der Limfang bes Serkes ift nidft unbedentemb - nadh bem Sian um etwa 35 Bogen - gewadyjen, uno zwar wiro fich bieje Erweiterung einerjeits burch Şinzufügung neuer Disziplinen, anorerjeits burch größeren

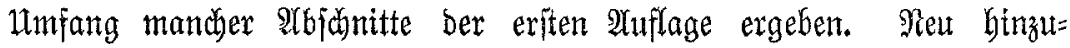

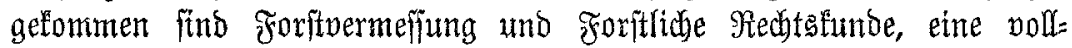

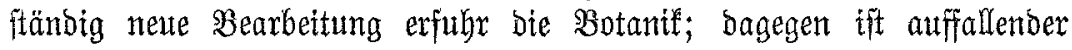

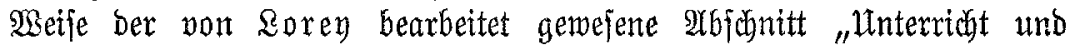

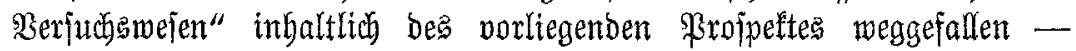
worum wohl?

Der ermeiterte Imfang Der netten AHflage gab Beranlafjung, bie=

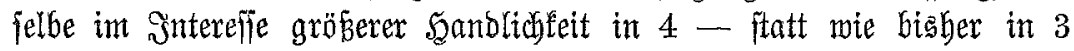
- Bänden exjbeinen zu laffen und den Stoff in etwas anderer und, wie wir glauben möchten, ridbtigerer $\mathfrak{B}$ eije zu gliebern. So murbe

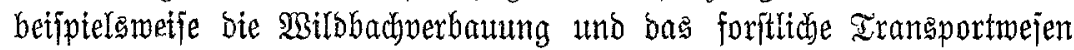

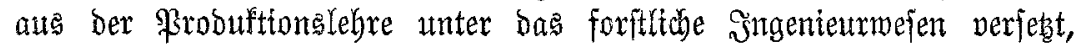

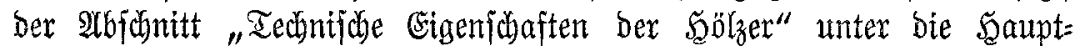
rubril "Frorttbenubung" eingereigt, Foritgejofitcte mit ber Foritnermaltung,

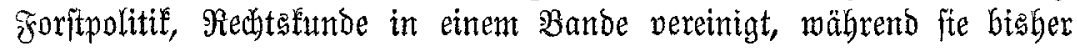

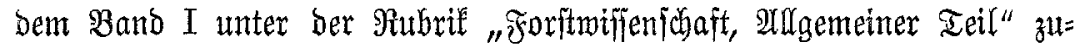
geteilt mar. -

Rideft geringe Rücten hat in bent langen 3ettraum von 15 Şabren

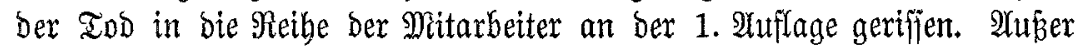
sorey felbft, ber jeond Durch den von ihm bereits polfitänbig bearbeiteten

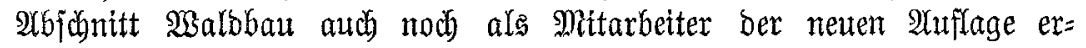

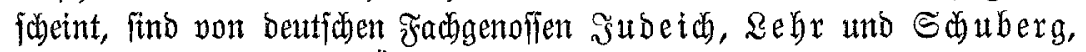
von Mitarbeitern atts Diterretc Frofeffor Exner=sBien, Foritmeifter

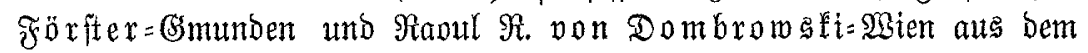

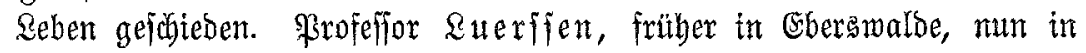
Räniggberg hat jeine Mitwirfintg (\$Botanie) bei ber Reuauflage zurüd:

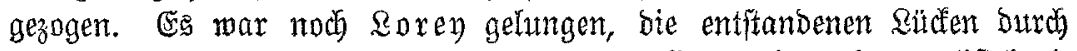

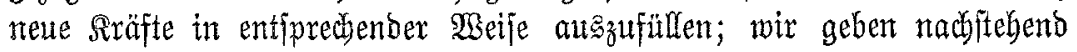




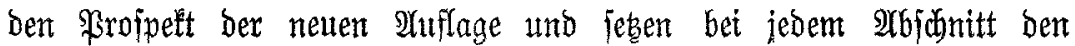
Mamen bes früheren \$earbeitere, injoferne berjelbe ein anderer war, in אlammer bei.

\section{Intand}

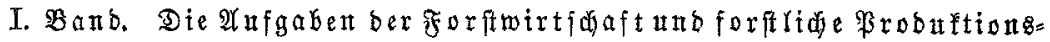
lebre, erfer $\mathfrak{F}$ etl.

1. Die Mufgaben ber Forfinirtitaft. - Frof. Dr. Weber=Miunden.

2. Forfttide Stanbort\$lefre. - Frof. Dr. Ramann-Mitncien.

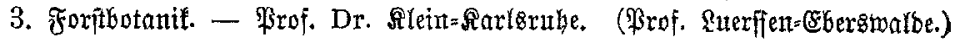

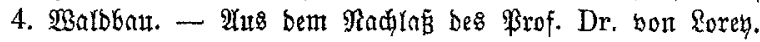

II. Banb. Forflitue Probuftionglebre, zweiter Teil.

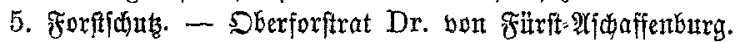

6. Forftbenukung.

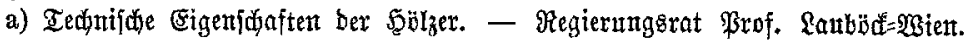
(\$rof. Erner=:Miten).

b) Forftrobuften=(Ernte, Berwertung unb 9 Unfbewahrung. - Steh. Sberforftrat Dr. Stoetser=Eijentat).

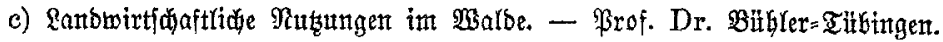

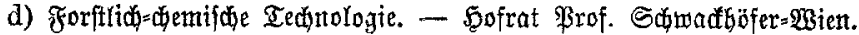

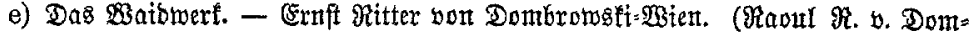
brow $\mathbf{f} t=2$ Biten).

f) Fifdjerei und Fijüztdt. - Beh. Regierungorat Prof. Dr. Mekger=Münben.

III. Band. Forftide Betriebslebre and forfities Sngenieuraefen.

7. Forftbermefingslehre. - Frof. Dr. Fromme=(Siefen. (Metr.)

8. Walbmertrednung uno Statif. - (5eh. Dberforftrat Dr. Stoeker=Eifentad. (Prof. \&ehr= Mitndidert.)

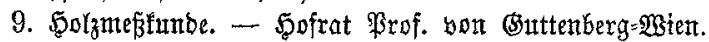

10. Forfeintidututg. - Seh. Dberforftrat Dr. Reumeifter=Tharand. (Skerforftrat Indeidis= Tharandt.)

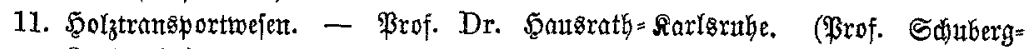
Rarlerrutie.)

12. WilbGadiverbanung. - Forftrat $\mathfrak{B r o f}$. Wang=Wien. (Forftmeifter Förfter. (Simunbett.)

IV. Band. Forftitue $\mathfrak{B}$ ermaltung unb Forftgefdidte.

13. Forftwerwaltungelehre. - Forftmetfer Prof. Dr. Sdiwaptadi $=$ (Sberstwalbe.

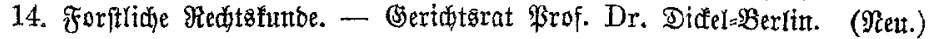

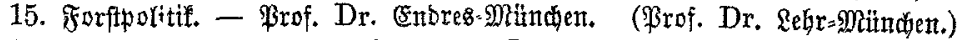

16. Forftgefouidte. - Forftmeifter Frof. Dr. Sdwappad)=(Ebersmalbe.

2lls eine weitere Ergänzung wird jedem Band ein augjübrlicbes alphabetijabes Sachregifter beigegeben, woburch ein rajd)es Rachjoblagen

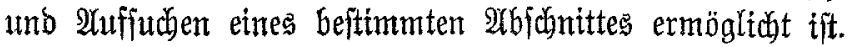

Das ganze umfangreide Werl wird in 25 Riejerungen à 6 Bogen ericheinen, fonach rund 150 Dructbogen umfaffen. Dex \$reis beträgt pro Rieferung $2 \mathscr{M}$, und wird ben Gsejamtbetrag bon $50 \mathscr{M}$ attd bann 


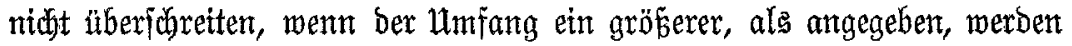
folte, während bet etwaigem geringeren Unmfang entipred)ende \$reis= ermäß̄igung eintreten würoe.

WBir befalten uns feinerzettge eingebendere Beriohterftattung vor.

Dr. Fưr rit.

\section{Rr. 30.}

\section{Die Fifheret als Nebenbetrieb bes Ranbwirtes unt forit=}

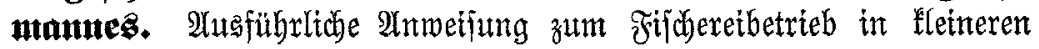

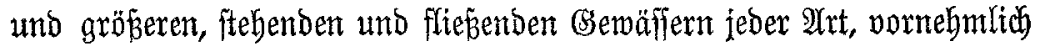
in Seen, Bächen, Sarpfen= uno Frorellented den, von Dr. Emil :

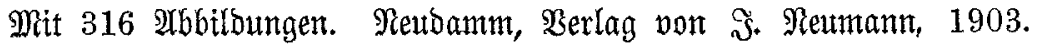
801 S., 扸is ungeb. $14 \mathscr{M}$.

Bu ben $\mathscr{H}$ ufgaben, weldhe an ben Forjtwirt neben jeiner Szaupt=

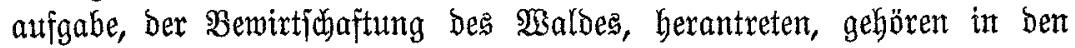

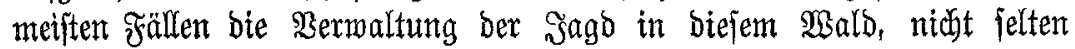
aber aud iene ber Fijheret in ber bieje Maldungen burchjtrömenten

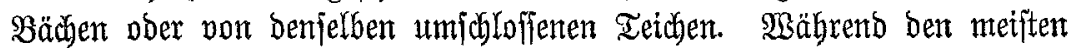
Foritbeamten aber bie nötigen jagditchen Renntnifje zur Seite ftehen, bie J̧ago von ihnen mit \&uịt und Riebe geübt wird, liegt mangels foldser

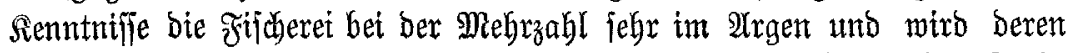

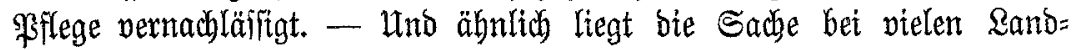
wirten, obwohl gerabe in gegenwärtiger Beit bte Ranowirte alle Ber=

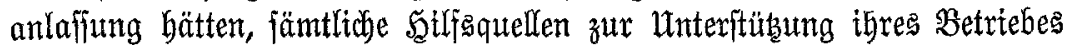
fliifitig zu machen.

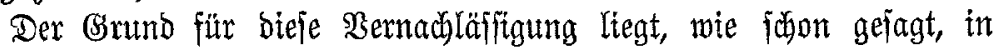

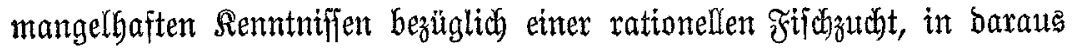

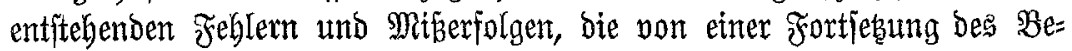
triebes abjajredfen. Bu foldhen Mijerfolgen führt es insbejombere, menn ber Beftber von Fifodgemäfiern an Stelle eimes einfachen aber lohnenden

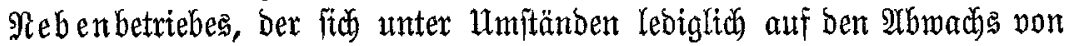

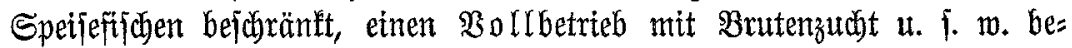
treiben will, für meldhen bie şebiutgungen nidbt gegeben fïtb, bie nötigen Renntnifje fehlent. - Fitr bie Ftideret als Rebenbetrieb, uno zwar bie zabme Filcheret in ablapharen Teichen, bann bie wilbe fijheret in größ̈eren Seen und fließ̧enden Gewäffern will ber פerfaffer in vorliegen=

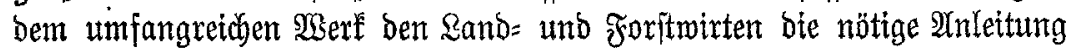
geber.

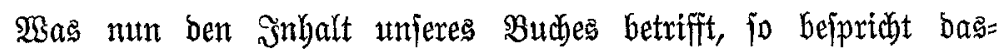




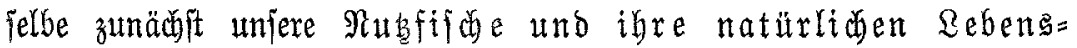
bebingungen, bierbei bie 2 miditigiten ârten, Rarpfen und rovellen, bejonders Gervorbebend. 3wei weitere Rapitel find ber I eichwirtidaft

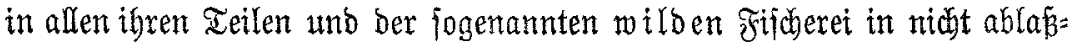
bàren Seen und fliebenden Gemäfjern gewibmet. Die Mittel bes inten= fiven Birtichaftsbetriebes: bie Mernidutung ber jogenannten bartent Flora, bie Bebandlung bes Bobens ber" abgelafienten Ieidhe, bie Diungung ber

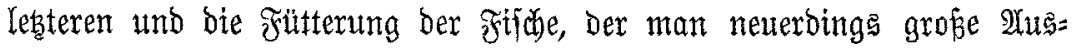
Defmnng gibt, finto Begenitano bes nächiten Ubichnitts, während bte folgenden ben Fifhfang, bie verjojiebenen Irten begfelben, bie $2 \mathbb{H} f=$ bemahrung und ben Serfand ber Fride behandeln und bierüber eingehende Belebrung geben. (Ebenfalls gründtche ßepprechung finden bie

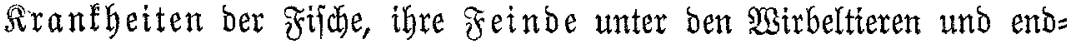

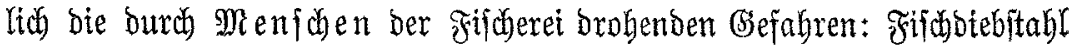

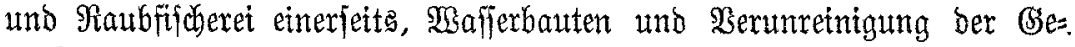
wäffer andererjetts. Den Schlup̃ endich bildet ein ber $\mathfrak{B}$ erwertung ber

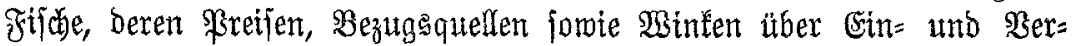
fauf gemibmeter 2 bij

Die vorftehenbe überficht gewährt einen Einblide in ben reiden Sn= balt bes mit firdtlicher Sorgfalt bearbeiteten unb mit zahlreichen guten

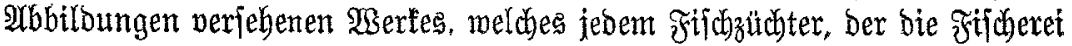
im Nebenbetrieb pflegt, reidje $\mathfrak{B}$ elehrung nadh allen Seiten bin zu gewähren vermag. Die 2lusitattung feitens ber $\mathfrak{B}$ erlagshanblung mutp als eine jefyr gute bezeidgnet merben.

\section{ఇr. 31.}

Praftijdjex 1tugeziejerfalenber. (sin $\mathfrak{B u h}$ für jebermann von Feinrich Frh. von Schillting. Mit 332 Drig.=8eidumungen bes Berfaffers. Franlfurt a. D., Berlag von Trowitida $\mathfrak{u}$. Sobn, 1902. 193 E. Frets geb. 3 M.

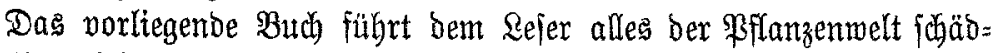
liche Ungeziefer, ebento aber aud alle Schmarober auf Menfden uno

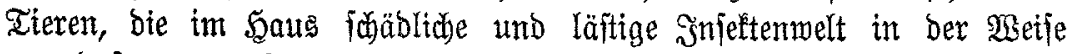
vor, baf es von Monat zu Monat fortichreitend bie in jebem beriflben auftretenden, als Earven ober Šmagines burd ibre Erjobeimung bezw. ben burch ite angeridteten Sdjaben in bie $\mathfrak{A}$ ugen fallenden Sinjeften in Wort und Bild barfteflt und in turzen 2 sorten ben burd fie angeridhteten Sdaben wie beffen \$efämpjung bejofreibt. Es will ber Berfaffer baburdh jebermann bie Möglidfeit zut rechtzettiger Exfenmung unb redftzeitigem 
Einjeffreiten geben, zur tumliduften Berminoerung ber oft fo bebentenden Şnjeltenjwäben beitragen.

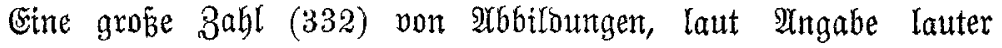
Driginalzeidamungen bes Berfaffers, ermöglidjen bie leidhte Ërfennung des

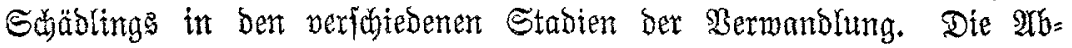
bifoungen find im ganzen recht gut, einzelne allerbings, jo jene ber Berre S. $29 \mathfrak{u}$. 68, ber Riejernipinnerraupe (Iannengludfe) S. 35 , bie auf

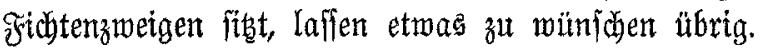

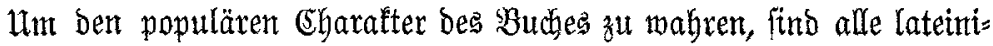
fiben Ramen weggelaffen, was bet ben lofal oft fo verjebiebenen und un=

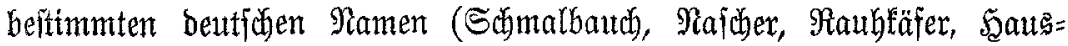

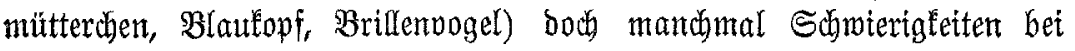
Der Beitimmung, bem Radbjhlagen verurfacten lam. So bätte z. $B$. bei Tannenglucfe wentigitens ber Rante "Riefernipinner", bei Tamnenlaus ber richtige Rame "richtenrinbenlaus" angegeben werden follen.

$\mathscr{D a} \tilde{\mathfrak{B}}$ auth Sdymetterlinge wie Totentopf, Dleanberidgwärmer,

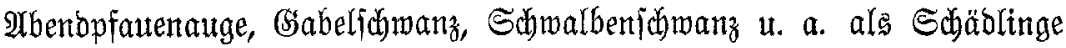
angefübrt find, bürfte boch etras za weit gehen.

šm übrigen vermag bas Buch aber feinten 3 medf, zur Renntnis ber

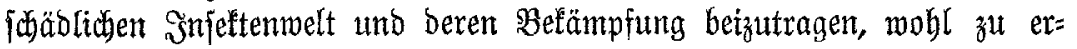
füllen und $\mathfrak{t a n n}$ baber allen Raien auf bem Gebiet ber Sonfeftentunde Ġärtnern, Blumenzüudtern, \&anbleuten, Şausfrauen wohl empfohlen werben.

F.

Nr. 32.

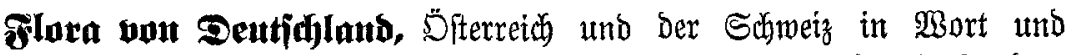
Bild, von $\mathfrak{B r o f e f i j o r ~ D r . ~} \mathfrak{I} \mathfrak{y}$ mé. Mit 616 Fflanzentafeln in zarben= brud und ca. 100 Bogen Iert. Bweite vermebrte und verbefferte 2luflage. Gera, Berlag von Frieor. von 8 ezfönib. Bollitändig in 56 Rieferungen à $1,25 \mathscr{M}$.

Wir baben im vorigen Sabr bas Ericheinen biefer netten IUffage, von melcher bamals bie betben eriten sieferungen vorlagen, angezeigt und über ßlan unb 2tueftellung bes umfangreiden Merfes furz berichtet.

Mittlermeile find 6 neue Rieferungen, Deren jebe 2 Bogen Text und 11 farbige Iafeln entyält, erfabienen. Wä̆hrend bie beiben erften \&iefe= rungen fith mit ben Siryptogamen und bezm. mit ben ₹arnen bejōäftigten - für alle übrigen zu Diejer Silaffe gehörigen nteberen Formen exjcheint eine Ěrgänżıng in 3 bejonders fäuflichen Bänden -, bebandeln bie 6 weiteren vor uns liegenden Rieferungen bie \$ihanerogamen, tnd zwar zunäbit bie (Symnoiperment, bie Soniferen und Grnetumgerwädje, un bam 
glt Den IInginfpermen und Deren 1. Slafie, Den Mentocotylen überzugehen.

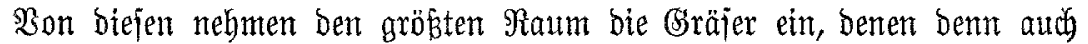

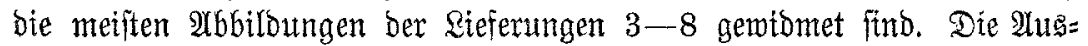
fübrung ber lebtern ift eine burchaus gute, fo baf jie bet Beftimmung ber einzelnen alrten gute Dienfte zu leiften vermögen; insbejondere gift leţteres von ben in nambafter Bergrößerung beigegebenen für bie Be ftimmung michtigen $\mathfrak{B l u ̈ t e n ~ u n d ~ B l u ̈ t e n t e i l e n . ~}$

Das $\mathfrak{B e r f}$ bürfte jebent, ber tiefer in bas Bebiet ber $\mathfrak{B o t a n i l}$ ein= bringen will, eine willfommene sabe jein.

\section{গx. 33.}

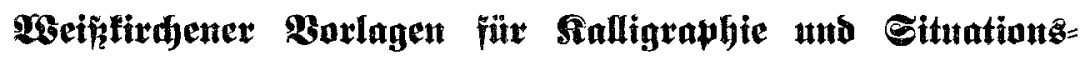

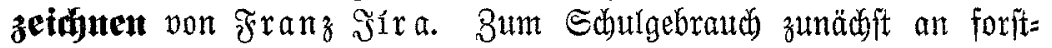

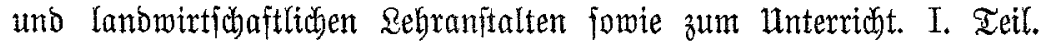
Salligraphte.

Der Berfaffer meifit im Sormort auf bie grofe Bebeutung einer ent= fprectenden Fertigfeit in ber Salligraphie und im Sttuationszeidunen im praftifichen forftlicben Betrieb him. Sn Beziehung auf ben z. 3. vor= liegenden I. Tetl, in bem ex uns auf 9 Tafeln hübid und fauber ge= zeidhnete Subbjitahenworlagen veridbiebener Schriftarten mit erläuternoem

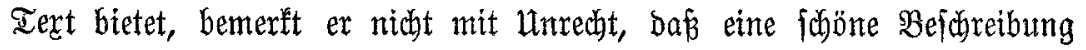
eine minber volfontmene 3eichnung bebt und gefällig madbt, währeno

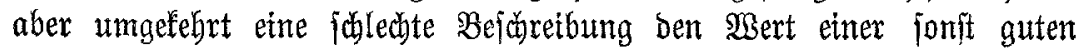
3eidjung wefentlich herabbrüdt.

Die $\mathfrak{B o r l a g e n}$ fönnen zum Unterridht mur empfoblen mexben.

D.

গr. 34.

Die Bixid mi Put=, Damt, Meh=, Srfwaxz= umb Gemswilb,

von Ernft Ritter von Dombrowgt. Mit 8 sollbtldern. Relt=

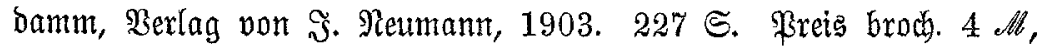
geb. $6 \mathscr{M}$.

Ein hervorragender $\mathfrak{B e i o n t a n n , ~ b e r ~ b e r e i t s ~ b u r c h ~ z a b l r e i d y e ~ j a g o l i d j e ~}$

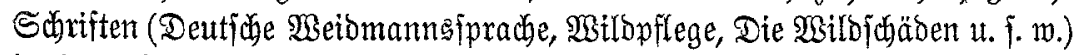
in Şägerfreifen wohl befamnt ift, bietet ber Sägermelt in dem vorliegenden

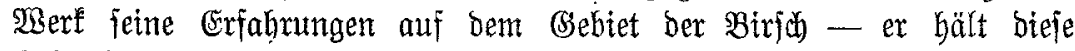
Schretbmeife für bie einzig richtige gegenüber ber fonft aud gebräuch)=

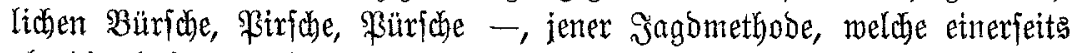
als bie bohe Schule ber Jagd bezeldnet wirb, andererjeits aber nach 


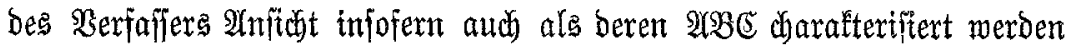
tann, als fie ben angehenden Şäger ganz auf eigene ơuñe ftellt, ben

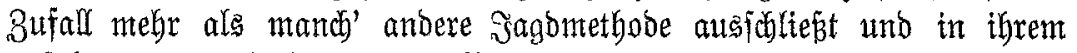
Erfolg bas sprobuft etres zielbemnipten und wohldurchdachten \$lanes Dariftellt.

Dombromsli bejpridyt zuterft bie allgemeinen Birfidyregeln, fobann

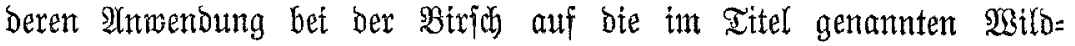

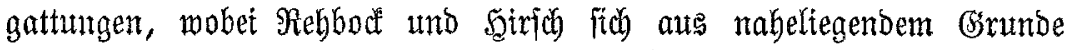
einer bejonders eingehenden Befprectung erfreuen. Dem Serfaffer war es vergönnt, bie Birich an ben verichtebentten Drten und atf tetl= weife ganz vorzäglichen Sagdrevieren, utnter jebr wecbjelnden Ierrain=

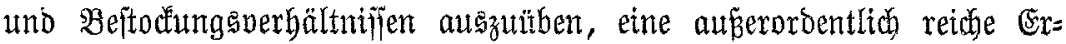
fabrung fteht ihm zur Seite und jo vermag er feine 2Uthlettungen mit meift eigenen Sagderlebnifjen retch zu ifuftrieren, bie settüre bes Buthes zul einer unterbaltenden und anregenden zut machen, vermag fowohl ben Yernbegiertgen $2 \mathfrak{A} \mathfrak{n}$ fänger wie ben älteren şäger zat befriebigent.

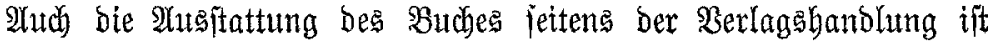
eine rectst gute uno wirb ebenfalfs bazat beitragen, Demjelben Freunbe in Şägerfretfen zat erwerben.

$\mathrm{F}$.

\section{Altatizent.}

Gohe Eiḑenholjpreife.

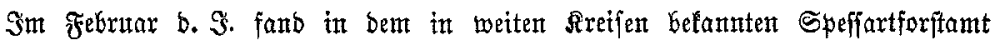

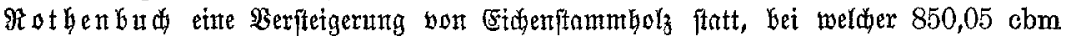

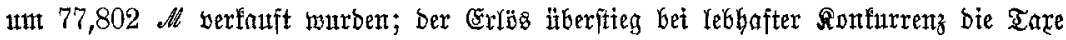

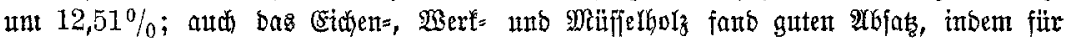

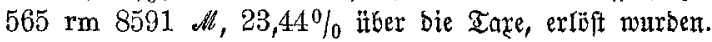

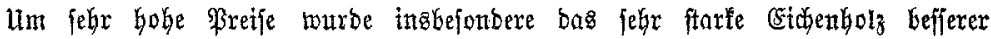

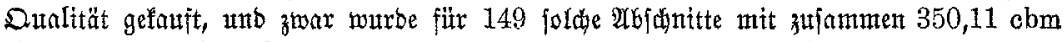

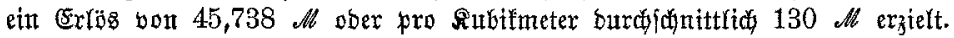

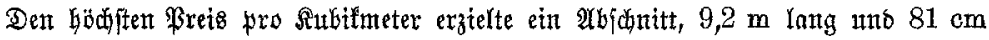

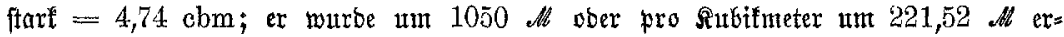

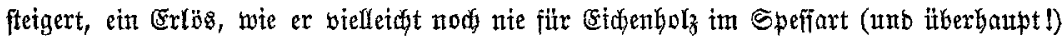

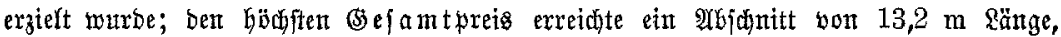

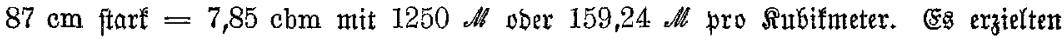

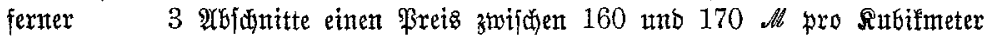

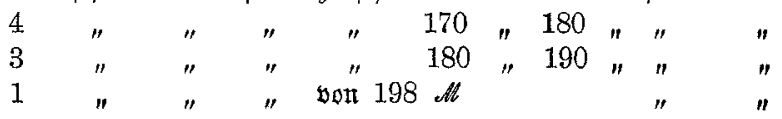

